\title{
THERE IS NO EXACTLY $k$-TO- 1 FUNCTION FROM ANY CONTINUUM ONTO [0,1], OR ANY DENDRITE, WITH ONLY FINITELY MANY DISCONTINUITIES
}

\author{
JO W. HEATH
}

\begin{abstract}
Katsuura and Kellum recently proved [8] that any (exactly) $k$-to1 function from $[0,1]$ onto $[0,1]$ must have infinitely many discontinuities, and they asked if the theorem remains true if the domain is any (compact metric) continuum. The result in this paper, that any (exactly) $k$-to- 1 function from a continuum onto any dendrite has finitely many discontinuities, answers their question in the affirmative.
\end{abstract}

1. Introduction. Continuous (exactly) $k$-to-1 maps have been extensively studied for decades. Much research has concentrated on which spaces can be the domain of such a map, and for which $k$ (see bibliography). As for which spaces can be the image of such a map, Harrold [5] showed that no arc could be (for any $k>1$ ), and he showed that if the domain is a simple graph, then the image contains a simple closed curve. Recently Nadler and Ward [12] proved that if the image $Y$ is locally connected, then there is a $k$-to-1 map onto $Y$ iff $Y$ contains a simple closed curve. They also proved that any continuum (locally connected or not) that contains a nonunicoherent subcontinuum is the image of a $k$-to-1 map.

If a discontinuity or two is allowed for the $k$-to-1 function, more spaces qualify for both domain and range, not surprisingly. For instance, K. Kuperberg [9] has constructed a 2-to-1 function on a disk with one discontinuity, and Kellum and Katsuura [8] showed that for $k$ odd or $k=4$, there is a $k$-to- 1 function from $[0,1]$ into $[0,1]$ with exactly one discontinuity. The author has shown in $[7]$ that if $k$ is even and $k>4$ then there is a $k$-to- 1 function from $[0,1]$ into $[0,1]$ with two discontinuities (and none with fewer than two), and has shown in [6] that every 2-to-1 function from $[0,1]$ to any Hausdorff space has infinitely many discontinuities. Kellum and Katsuura also showed that if the image is compact, then any function from $[0,1]$ to $[0,1]$ requires infinitely many discontinuities for $k>1$. In the same paper [8], Kellum and Katsuura ask if every $k$-to-1 function from any continuum onto $[0,1]$ must have infinitely many discontinuities. The main result of this paper is that any (exactly) $k$-to-1 function from any continuum onto a locally connected continuum with no simple closed curve (a dendrite) has infinitely many discontinuities. This answers the Katsuura-Kellum question in the affirmarive.

Requiring no simple closed curve in the image is clearly necessary since otherwise there is a continuous map [12]. In view of Nadler and Ward's similar result with nonunicoherent subcontinua, the following seems a natural question:

Received by the editors September 15, 1986. Presented at the Atlanta AMS meeting January 8, 1988.

1980 Mathematics Subject Classification (1985 Revision). Primary 54C10, 26A15, 26A03.

$K e y$ words and phrases. $k$-to-1 function, $k$-to-1 map. 
Question. Is there a $k$-to- 1 function from any continuum onto any arc-connected, hereditarily unicoherent continuum with only finitely many discontinuties?

In the Katsuura-Kellum result mentioned above, compactness of the image is crucial, and it is for this paper's result also, as the following simple example demonstrates:

EXAMPLE. Let $X$ be the dendrite in the plane that is the union of straight line segments with one endpoint at the origin $(0,0)$ of length $1 / i$ and slope $i$ for each positive integer $i$, and let $Y=[0,1)$. Define $f((0,0))=0$ and divide the countably many half open, half closed intervals left in $X-\{(0,0)\}$ into $k$ disjoint infinite collections, $G_{1}, G_{2}, \ldots, G_{k}$. For each $i<k$, map the first arc in $G_{i}$ homeomorphically to $[0,1 / 2)$, the second to $[1 / 2,3 / 4)$, etc. Map the first arc in the last collection $G_{k}$ to $(0,1 / 2]$, the second to $(1 / 2,3 / 4]$, etc. Then $f$ is $k$-to- 1 and is discontinuous only at $(0,0)$.

2. Some definitions. A continuum is a compact connected metric space.

A function is $k$-to-1 if each point in the image has exactly $k$ inverse points.

A dendrite is a locally connected continuum containing no simple closed curve.

A sequence of sets is null if their diameters converge to 0.

The arc $A$ is irreducible from $p$ to $S$ if one endpoint of $A$ is the point $p$, the other endpoint of $A$ lies in the set $S$, and no point of $A$ other than the two endpoints lies in $S$.

3. Preliminary lemmas. Some version of Lemma 0 was independently noticed by Kellum and Katsuura.

LEMMA 0 . If $f$ is a finite-to-one function from the compactum $X$ to the dendrite $Y$ with only finitely many discontinuities, then $X$ does not contain an infinite collection of disjoint continua whose diameters are bounded away from 0.

PROOF. Suppose there is such a collection of disjoint continua. Then there is a subsequence $\left\{C_{i}\right\}$ that converges to the nondegenerate continuum $C^{\prime}$. Since there are only finitely many discontinuities, $C^{\prime}$ contains a nondegenerate continuum $C$ that contains no discontinuity for $f$, and since all of $C$ cannot map to the same point, there are points $r$ and $s$ in $C$ that map to different points in $Y$. Let $t$ denote an interior point of the arc from $f(r)$ to $f(s)$ in $Y$; then $t$ is a cut point of $Y$ and there are open sets $R$ and $S$ containing $f(r)$ and $f(s)$ so that any continuum in $Y$ that intersects both $R$ and $S$ also contains $t$.

If $i$ is large enough then $C_{i}$ contains no discontinuity of $f$ and contains points close enough to $r$ and $s$ that $f\left(C_{i}\right)$ is a continuum in $Y$ that intersects both $R$ and $S$, and hence contains $t$. This makes infinitely many point inverses for $t$ since the $C_{i}$ are disjoint, a contradiction.

COROLlaRY 1 TO LEMMA 0 . If $f$ is a finite-to-1 function from the continuum $X$ to the dendrite $Y$ with only finitely many discontinuities, then $X$ is locally connected and hence arc-wise connected.

COROLLARY 2 TO LEMMA 0 . The statement of Lemma 0 remains true if $Y$ is a continuum with the property that each pair of points in $Y$ is separated by some finite subset of $Y$. 
COROLLARY 3 TO LEMMA 0 . If $f$ is a finite-to-1 function from a compactum to a dendrite $Y$ with finitely many discontinuities, and if $[a, b]$ is an arc in $X$, then $\lim \{f(x) \mid x \in[a, b], x \rightarrow b\}$ exists.

PROOF. Otherwise there is a sequence of disjoint subarcs in $[a, b]$ converging to $b$ whose left endpoints map to a sequence in $Y$ that converges to the point $s$ in $Y$ and whose right endpoints' images converge to a different point $r$ in $Y$. As in the proof of Lemma 0 , the images of the disjoint subarcs map arbitrarily close to both $r$ and $s$ and hence most of the arcs map to a point $t$ in the arc from $r$ to $s$, yielding the same contradiction. Q.E.D.

Lemma 1 essentially reduces the theorem to the case where $Y$ is an arc although that may not be apparent yet. It has as a corollary that if all of the discontinuities of $f$ map to two points in $Y$ and $A$ is the arc between them, then $f^{-1}(A)$ is a continuum so that $f \nmid f^{-1}(A)$ has all of $f$ 's properties and maps onto an arc. (To see that $f^{-1}(A)$ is connected, let $a$ and $b$ be any two points of $X$ that map to $A$ and note that an arc in $X$ from $a$ to $b$ maps to $A$ by Lemma 1 so that $a$ and $b$ are arc-connected in $f^{-1}(A)$.) The fact that the discontinuities are probably more widely dispersed will cause some difficulties, but there will still be an arc $A$ in $Y$ where $f \backslash f^{-1}(A)$ yields a contradiction.

LEMMA 1. Suppose that $f$ is a $k$-to-1 function from the continuum $X$ onto the dendrite $Y$ whose set of discontinuities, DIS, is finite, that $E^{\prime}$ is a 1-complex in $Y$ containing $f(\mathrm{DIS})$, and that $[x, y]$ is an arc in $X$ with $f(x)$ and $f(y)$ both in $E^{\prime}$. Then $f([x, y])$ is a subset of $E^{\prime}$.

Proof. Let $E$ denote $f^{-1}\left(E^{\prime}\right)$. Since DIS $\subset E$ and $E^{\prime}$ is compact, $E$ is closed in $X$ and contains both $x$ and $y$ by assumption. Hence $[x, y]-E$ has an open component $(a, b)$, unless $f([x, y]) \subset E^{\prime}$ as desired. Since $(a, b)$ is connected and misses DIS, its image $f((a, b))$ is connected and lies in some component $C$ of $Y-E^{\prime}$. Let $A$ be an irreducible arc from some point of $f((a, b))$ to $E^{\prime}$, which connects $f((a, b))$ to $E^{\prime}$ on the off chance that $a$ and $b$ are both in DIS and $\mathrm{Cl}(f((a, b)))$ is in $C$. If $\mathrm{Cl}(f((a, b)))$ intersects $E^{\prime}$, let $A=\varnothing$.

Since every subcontinuum of a dendrite is a dendrite, $D=A \cup \mathrm{Cl}(f((a, b)))$ is a dendrite with one endpoint $e$ in $E^{\prime}$ and another $q_{0}$ not in $E^{\prime}$. Every dendrite has at least two endpoints and if $D$ had two in $E^{\prime}$ then there would be two arcs between them in $Y$, one in $D$ and one in $E^{\prime}$, not possible since dendrites are uniquely arcwise connected.

The initial dendrite $D$ will be augmented with images of special arcs (defined below as "eligible") getting larger dendrites at each ordinal stage because $f$ is $k$ to-1. The contradiction is that the process does not end and also cannot become uncountable. $E$.

DEFINITION. The $\operatorname{arc}[w, z]$ in $X$ is eligible from $w$ if $[w, z)$ misses $E$ and $z$ is in

DEFINITION. The eligible arc $[w, z]$ from $w$ extends the dendrite $D^{\prime}$ through its endpoint $q$ if $f(w)=q$, and for some subarc $\left[w, x^{\prime}\right]$ of $[w, z), q$ separates $f\left(\left[w, x^{\prime}\right]\right)-$ $\{q\}$ from $e$ in $Y$. The dendrite $D^{\prime} \cup \mathrm{Cl}(f([w, z)))$ is the extension.

(All of the dendrites that will be considered will contain the special endpoint $e$ in $E^{\prime}$ described before.) 
We will extend the dendrite $D=A \cup \mathrm{Cl}(f((a, b)))$ first. Recall that $q_{0}$ is an endpoint of $D$ not in $E^{\prime}$ and that $e$ is the endpoint of $D$ in $E^{\prime}$. Denote by $B$ the arc from $q_{0}$ to $e$ in $E^{\prime}$. Let $w_{1}, w_{2}, \ldots, w_{k}$ be the $k$ points in $X-E$ that map to $q_{0}$, and let $U_{1}, U_{2}, \ldots, U_{k}$ be disjoint open sets in $X-E$ containing them that map into $C$ with the further property that if $w_{i}$ is not in $[a, b]$ then $U_{i}$ also misses $[a, b]$. Since $X$ is arc-connected by Lemma 0 there is for each $i$ an eligible arc $R_{i}$ from $w_{i}$ and a subarc $R_{i}^{\prime}$ in $U_{i}$ with endpoint $w_{i}$.

We wish to show that one of the $R_{i}$ arcs extends $D$ through $q_{0}$, where $R_{i}^{\prime}$ satisfies the separation property. So suppose that for each $i$ there is a point $x_{i}$ in $R_{i}^{\prime}$ such that the arc $T_{i}$ from $f\left(x_{i}\right)$ to $e$ does not contain $q_{0}$. Since $f\left(R_{i}^{\prime}\right)$ contains an arc from $q_{0}$ to $f\left(x_{i}\right), T_{i}$ connects $f\left(x_{i}\right)$ to $e$, and $B$ is an arc from $q_{0}$ to $e, B \subset f\left(R_{i}^{\prime}\right) \cup T_{i}$. Since $q_{0}$ is not in $T_{i}$, some initial segment $B_{i}=\left[q_{0}, q_{i}\right]$ of $B$ is contained in $f\left(R_{i}^{\prime}\right)$. Let $N=\bigcap\left\{B_{i} \mid i=1,2, \ldots, k\right\}$, say $N=\left[q_{0}, q^{\prime}\right]$. Each point of $N$ has a point inverse in $R_{i}^{\prime}$ and there are $k$ disjoint $R_{i}^{\prime}$ arcs, so all of the point inverses of $N$ are accounted for in the union of the $R_{i}^{\prime}$ arcs.

At this point the argument for the initial dendrite differs from that of later dendrites. If $w_{i}$ belongs to the arc $(a, b)$ then both $\left[w_{i}, a\right]$ and $\left[w_{i}, b\right]$, subarcs of $[a, b]$, are eligible from $w_{i}$ since $a$ and $b$ are in $E$ and $(a, b)$ misses $E$. One of them, say $\left[w_{i}, a\right]$, satisfies $f\left(\left[w_{i}, a^{\prime}\right]\right) \cap N=\left\{q_{0}\right\}$ for some $a^{\prime}$ in $\left(w_{i}, a\right]$, since each point of $N$ has only one point inverse in each $U_{i}$ and since $\left[w_{i}, a^{\prime}\right] \cap\left[w_{i}, b^{\prime}\right]=\left\{w_{i}\right\}$. But this means that $\left[w_{i}, a\right]$ extends $D$ through $q_{0}$. So we may assume that no $w_{i}$ is in $(a, b)$.

Since each point inverse of $q_{0}$ misses $(a, b), q_{0}$ is not in $f((a, b))$, so $q_{0}=$ $\lim \{f(x) \mid x \in(a, b), x \rightarrow a\}$, or $b$ of course, from the Corollary to Lemma 0 . No $w_{i}$ is $a$ or $b$ since $a$ and $b$ are in $E$ so each $w_{i}$ is not in $[a, b]$ and the open set $U_{i}$ containing $w_{i}$ misses $[a, b]$. The irreducible arc $A$, if nonempty, has one endpoint $e$ in $E^{\prime}$ and the other endpoint in $f((a, b))$ which cannot be an endpoint of the union $D=A \cup \mathrm{Cl}(f((a, b)))$, so $q_{0}$ is not in $A$. This means that the $\operatorname{arc} B$ from $q_{0}$ to $e$ has an initial segment in $f((a, b)) \cup\left\{q_{0}\right\}$, so $N$ must intersect $f((a, b))$. This is a contradiction since each point inverse of $N$ is in some $U_{i}$ and every $U_{i}$ misses $[a, b]$.

Therefore for some least $i, q_{0}$ separates $f\left(R_{i}^{\prime}\right)-\left\{q_{0}\right\}$ from $e$. Let $e^{\prime}$ denote the endpoint of $R_{i}$ in $E$. Define $D_{1}=D \cup \mathrm{Cl}\left(f\left(R_{i}-\left\{e^{\prime}\right\}\right)\right)$. Note that the larger $R_{i}$ is used, and note that $\mathrm{Cl}\left(f\left(R_{i}\right)\right)-f\left(R_{i}\right)$ is a single point, or is empty, from the Corollary to Lemma 0.

Now suppose that $D_{\alpha}$ has been constructed for each $\alpha<\beta$ so that:

(1) $D_{\alpha}$ is a dendrite in $C \cup\{e\}$ containing $D$,

(2) if $q$ is any endpoint of $D_{\alpha}$ other than $e$, then there is an eligible $\operatorname{arc}$ in $X$ that extends $D_{\alpha}$ through $q$,

(3) if $\alpha$ is not a limit ordinal then there is an eligible arc $[w, z]$ that extends $D_{\alpha-1}$ through some endpoint of $D_{\alpha-1}$ and $D_{\alpha}=D_{\alpha-1} \cup \mathrm{Cl}(f([w, z)))$, and

(4) if $\alpha$ is a limit ordinal, then $D_{\alpha}=\mathrm{Cl}\left(\bigcup\left\{D_{\gamma} \mid \gamma<a\right\}\right)$.

So far $D_{0}=D$ satisfies (1), (2), and (3), and $D_{1}$ satisfies (1) and (3). We will construct $D_{\beta}$.

First, assume that $\beta-2$ exists. There is an eligible arc $[w, z]$ that extends $D_{\beta-2}$, and $D_{\beta-1}=D_{\beta-2} \cup \mathrm{Cl}(f([w, z)))$. The dendrite $D_{\beta-1}$ has an endpoint $q$ in $C$ since it cannot have two endpoints in $E^{\prime}$ (lest $Y$ have a simple closed curve). Choose the endpoint $q$ in the new part $\mathrm{Cl}(f([w, z)))$. As before there are $k$ points $w_{1}, \ldots, w_{k}$ in $X-E$ that map to $q$, disjoint open sets $U_{1}, \ldots, U_{k}$ containing them that map 
into $C$ and whose closures are in $X-E$. For each $i$ there is an eligible arc from $w_{i}, R_{i}$, and a subarc $R_{i}^{\prime}$ in $U_{i}$ with endpoint $w_{i}$. Again, if, for each $i, q$ does not separate $f\left(R_{i}^{\prime}\right)-\{q\}$ from $e$, then there is an arc $N=\left[q, q^{\prime}\right]$ in the arc from $q$ to $e$ such that each point of $N$ has a point inverse in each $R_{i}^{\prime}$.

Now suppose that some $w_{i}$ is in $[w, z)$. Since the new endpoint $q$ is not the old, $f(w), w_{i}$ is in $(w, z)$. But $f\left(\left[w, w_{i}\right]\right)$ contains an arc from the old endpoint to $q$ and must then map onto $N$. This means the eligible arc $\left[w_{i}, z\right]$ has no point in $U_{i}$ other than $w_{i}$ that maps to $N$, so $\left[w_{i}, z\right]$ extends $D_{\beta-1}$. Hence we will assume that no $w_{i}$ is in $[w, z)$.

Thus $q$ is not in $f([w, z))$, so $q=\lim \{f(x) \mid x \in[w, z), x \rightarrow z\}$. But this means there are points of $[w, z)$ arbitrarily close to $E$ that map to $N$, contradicting the fact that each $\mathrm{Cl}\left(U_{i}\right)$ misses $E$ and the $U_{i}$ have all the point inverses of $N$.

Thus, for some least $i\left[w_{i}, z_{i}\right]$ extends $D_{\beta-1}$ through $q$; define $D_{\beta}=D_{\beta-1} \cup$ $\mathrm{Cl}\left(f\left(\left[w_{i}, z_{i}\right)\right)\right)$. Then properties (1) and (3) hold for $D_{\beta}$. We proved that the new endpoints of $D_{\beta-1}$ extend, and the old ones do by induction. Hence $D_{\beta-1}$ also satisfies (2).

Now suppose that $b-2$ does not exist, that is that $\beta-1$ is a limit ordinal and $D_{\beta-1}=\operatorname{Cl}\left(\bigcup\left\{D_{\gamma} \mid \gamma<\beta-1\right\}\right)$. If the endpoint $q$ of $D_{\beta-1}$ is in some $D_{\gamma}$ for $\gamma<\beta-1$ then some eligible arc extends $D_{\beta-1}$ through $q$ by the induction hypothesis. So assume $q$ is an endpoint of $D_{\beta-1}$ that is not in any previous $D_{\gamma}$. Exactly as in the nonlimit case, there is an arc $N=\left[q, q^{\prime}\right]$ in the arc from $q$ to $e$ and $k$ disjoint open sets $U_{1}, \ldots, U_{k}$ whose closures miss $E$ such that $f^{-1}(N)$ lies in the union of the $U_{i}$ open sets.

Each subarc $\left[q, q^{\prime \prime}\right]$ of $N$ has a point $f(x)$ that is from some previous extension, i.e. $x$ belongs to some eligible arc that extended some $D_{\gamma}$ for $\gamma<\beta-1$. Otherwise, the arc $\left[q, q^{\prime \prime}\right]$ is in $\lim _{\beta-1}=D_{\beta-1}-\bigcup\left\{D_{\gamma} \mid \gamma<\beta-1\right\}$. Since $Y$ is locally connected there is an ordinal $\gamma 1$ with points of $D_{\gamma 1}$ close enough to $q$ and $q^{\prime \prime}$ that there are small disjoint arcs connecting each of $q$ and $q^{\prime \prime}$ to $D_{\gamma 1}$. But $\left[q, q^{\prime \prime}\right]$ in $\lim _{\beta-1}$ misses the arc in $D_{\gamma 1}$ connecting the small arcs, and a simple closed curve is formed in $Y$. Hence $\lim _{\beta-1}$ contains no arc.

Hence there is a sequence of points $\left\{f\left(x_{i}\right)\right\}$ in $N$ converging to $q$ such that each $x_{i}$ is in an eligible arc. Since $q$ belongs to no previous $D_{\gamma}$, the eligible arcs can come from different extensions and hence are disjoint. But each contains a point of $E$. Since the sequence is null, it converges to some point of $E$, and eventually the $x_{i}$ are outside of the $\mathrm{Cl}\left(U_{i}\right)$ and map to $N$; a contradiction.

Thus all of the endpoints of $D_{\beta-1}$ extend, and $D_{\beta}$ can be defined as $D_{\beta-1}$ plus one of the extensions.

This completes the induction and Lemma 1 is proved.

The following real analysis lemmas will be needed later:

LEMMA 2. Suppose that $f$ is a continuous map from $(0,1)$ to $[0,1]$ that is at most $k$-to-1. Then there is a subinterval of $(0,1)$ on which $f$ is 1-to-1.

ProOF. Let $c$ be one of the values in $[0,1]$ with the maximum number, $j$, of point inverses, $j \leq k$. If $a_{1}, a_{2}, \ldots, a_{j}$ are the points that map to $c$ subscripted so that $a_{i}<a_{i+1}$, then the graph between $a_{i}$ and $a_{i+1}$ is either a hill (i.e. $f(x)>c$ for $x$ between $a_{i}$ and $a_{i+1}$ ), or a valley. Let $m$ be the number of hills and $n$ the 
number of valleys. Then $m+n=j-1$. Suppose $m \geq n$. Let

$$
L=\min _{i}\left\{\operatorname{lub}\left\{f(x) \mid a_{i}<x<a_{i+1}\right\}\right\}
$$

Suppose the graph over $\left(a_{1}, a_{2}\right)$ is a hill. If $f$ is not 1-to-1 over $\left(a_{1}, b_{1}\right)$ where $b_{1}$ is the least point of $\left(a_{1}, a_{2}\right)$ that maps to $L$, then some horizontal line $\{y=r\}$ with $c<r<L$ intersects the graph of $f$ at least three times between $a_{1}$ and $b_{1}$. This same line intersects the graph between $a_{1}$ and $a_{j}$ at least $2 m+2$ times. But $2 m+2 \geq m+n+2=j+1>j$, contradicting the maximality of $j$.

LEMMA 3. If $f$ is a finite-to-1 continuous map from $[0,1)$ onto $[0,1)$, then there is a collection $C$ of disjoint half open, half closed arcs in the domain $[0,1)$ such that $f$ restricted to $[0,1)-\bigcup C$ is $1-$ to- 1 and still onto $[0,1)$.

PROOF. Let $x_{1}$ be the largest number in $[0,1)$ that maps to 0 . If $x_{1}$ is not 0 , let $I_{1}$, the first member of $C$, be $\left[0, x_{1}\right)$ and let $f_{1}$ be the restriction of $f$ to $\left[x_{1}, 1\right)$. If $x_{1}=0$, set $f_{1}=f$. Well order the rational numbers in $(0,1), r_{1}, r_{2}, \ldots$ If $f_{1}^{-1}\left(r_{1}\right)$ has more than one point, let $s_{1}$ denote the least and $t_{1}$ the greatest. Put $I_{2}=\left[s_{1}, t_{1}\right)$ in $C$ and denote by $f_{2}$ the restriction of $f_{1}$ to $\left[x_{1}, s_{1}\right) \cup\left[t_{1}, 1\right)$. If $f^{-1}\left(r_{1}\right)$ has only one point, let $f_{2}=f_{1}$.

Note that since $f([0,1))$ has no maximum, $\lim \{f(x) \mid x \rightarrow 1\}=1$. Hence $f_{2}^{-1}\left(r_{2}\right)$ either lies in $\left[0, s_{1}\right)$ or it lies in $\left[t_{1}, 1\right)$. If more than one point in the domain of $f_{2}$ maps to $r_{2}$, let $s_{2}$ denote the least and $t_{2}$ the greatest. Again add $I_{3}=\left[s_{2}, t_{2}\right)$ to $C$ and note that the three elements of $C$ are disjoint. Call $f_{3}$ the restriction of $f_{2}$ to its domain with $I_{3}$ removed. If only one point maps to $r_{2}$ via $f_{2}$ let $f_{3}=f_{2}$.

In this way, maps $f_{1}, f_{2}, \ldots$ are constructed so that:

(1) $f_{j}^{-1}\left(r_{i}\right)$ has only one point for $i<j$,

(2) the image of $f_{j}$ is $[0,1)$,

(3) $f_{j}$ is either $f_{j-1}$ or there is a half open, half closed interval $I_{j}$ in the domain $D$ of $f_{j-1}$ so that $f_{j}$ is $f_{j-1}$ restricted to $D-I_{j}$, and

(4) if $y$ is any number in $[0,1)$ then every $x$ in the domain of $f_{j}$ that maps to $y$ lies in the same component of the domain of $f_{j}$.

Let $g$ denote the intersection of $\left\{f_{i} \mid i=1,2, \ldots\right\}$, and let $y$ be any number in the image $[0,1)$. We will show that there is exactly one $x$ in the domain of $g$ that maps to $y$.

First, there is at least one $x$ in the domain of $g$ that maps to $y$. For each of the finitely many $x$ in $\left[0,1\right.$ ) that maps to $y$ (via $f$ ), let $i_{x}$ be the least positive integer such that $x$ is not in the domain of $f_{i x}$ (if there is one). Let $j$ be the largest of these $i$ 's. Then since $f_{j}$ is onto, some $x$ las no $i_{x}$ and this $x$ will be in the domain of $g$.

Second, there cannot be two numbers, say $x$ and $w$, in the domain of $g$ that map to $y$. If so, then from prperty (4) above, the segment $[x, w]$ must be in a component of the domain of every $f_{i}$ since $x$ and $w$ are. But $f$ cannot be constant on $[x, w]$ so there is some rational number $r_{j}$ such that two numbers between $x$ and $w$ map to $r_{j}$. Since those two numbers are not both in the domain of $f_{j+1}$, the interval $[x, w]$ is not in the domain of $f_{j+1}$ after all. A contradiction. 


\section{Theorem and proof.}

THEOREM. If $f$ is a $k$-to-1 function from the continuum $X$ onto the dendrite $Y$ and $k>1$, then $f$ must have infinitely many discontinuities.

PROOF. Suppose on the contrary that the set DIS of discontinuities of $f$ is finite. Let $E^{\prime}$ be a minimal 1-complex containing $f(\mathrm{DIS})$ and let $J$ be $f(\mathrm{DIS})$ plus the junction points of $E^{\prime} . J$ is finite with, say, $n$ elements, so $E^{\prime}-J$ has $n-1$ open arcs, $A_{1}, A_{2}, \ldots, A_{n-1}$. Let $D=f^{-1}(J)$; then $D$ has $k n$ points.

From Lemma $0, X$ is arc-connected, so there is an arc, $B_{1}$, between two points of $D$ that otherwise misses $D$, and a second irredubible arc $B_{2}$ from a third point of $D$ to $B_{1}$, etc., getting a 1-complex $E$ such that: $E-D=\bigcup\left\{\operatorname{Int}\left(B_{i}\right) \mid i=\right.$ $1,2, \ldots, k n-1\}$. Since $D$ maps to $E^{\prime}$, it follows from Lemma 1 that all of $E$ maps to $E^{\prime}$.

Since $D=f^{-1}(J)$ and each $\operatorname{Int}\left(B_{i}\right)$ misses $D$ and is connected, each $\operatorname{Int}\left(B_{i}\right)$ maps into some $A_{j}$. There are $k n-1 B$ 's, $n-1 A$ 's, and $k>1$, so at least $k+1$ of the $B$ 's, say $B_{1}, B_{2}, \ldots, B_{k+1}$, map to the same $A$, say, $A_{1}$.

We will be primarily interested in those points of $X$ that map to $A_{1}$. If $p$ maps to $A_{1}$ and $T$ is an arc from $p$ to $E$, then $\operatorname{Int}(T)$ maps to $A_{1}$ too, by Lemma 1 again. Let $X^{\prime}=D \cup f^{-1}\left(A_{1}\right)$. Then:

(1) $f$ is $k$-to- 1 on $X^{\prime}$ to $J \cup A_{1}$,

(2) $X^{\prime}$ is compact, and

(3) if $p$ is in $X^{\prime}-D$, there is an arc in $X^{\prime}$ from $p$ to $D$.

MAIN CLAIM (proof later). $X^{\prime}-\left(D \cup B_{1} \cup \cdots \cup B_{k+1}\right)$ is the union of disjoint open arcs and half open, half closed arcs.

Let $M$ denote the collection of arcs from the main claim plus the interiors of the $B_{i}$ arcs, $i=1,2, \ldots, k+1$. Then $M$ decomposes $X^{\prime}-D$. For each open $\operatorname{arc}$ in $M$ there is an open sub arc on which $f$ is 1-to-1 (Lemma 2). Change the composition of $M$ by replacing each of its open arcs with an open arc on which $f$ is 1-to-1 plus the 0,1 or 2 leftover half open, half closed arcs. $M$ still covers $X^{\prime}-D$ and has at least $k+1$ open arcs, which will be denoted $R_{1}, R_{2}, \ldots, R_{k+1}$, with the subscripts arranged so that if $A_{1}$ is identified with $[0,1]$, then $\operatorname{glb} f\left(R_{i}\right) \leq \operatorname{glb} f\left(R_{i+1}\right)$ for $i=1,2, \ldots, k$. For bookkeeping purposes, color the open arcs in $M$ blue and the half open, half closed arcs black.

For each $i=1,2, \ldots, k$, disjoint subsets $G_{i}$ of $X^{\prime}-D$ will be constructed so that $f$ maps $G_{i}$ onto (glb $f\left(R_{i}\right), 1$ ) in $A_{1}$, and so that $G_{i}$ misses $R_{k+1}$. The contradiction will be that each point of $f\left(R_{k+1}\right)$ has one inverse in $R_{k+1}$ and $k$ others in the $G$ 's, one too many for a $k$-to-1 function.

Construction of the $G_{i}$. Each $G_{i}$ will contain $R_{i}$ and black points from the half open, half closed $\operatorname{arcs}$ of $M$, and when $G_{i}$ is constructed its points will be colored green to distinguish them from the other points in $M$. Since the construction of $G_{1}$ is similar to any other except that there are no green points yet, we will start with the inductive step:

Suppose $G_{i}$ has been constructed for each $i<j$, so that:

(1) $f\left(G_{i}\right)=\left(\operatorname{glb} f\left(R_{i}\right), 1\right)$

(2) $f$ is 1-to-1 on $G_{i}$,

(3) $G_{i}$ is a subset of the union of the black arcs in $M$ plus $R_{i}$,

(4) $G_{i} \cap G_{n}=\varnothing$ if $i \neq n$, and 
(5) the collection $M$ is altered but still has $R_{n}$ for $n \geq j$, covers the portion of $X^{\prime}-D$ not in $\bigcup\left\{G_{i} \mid i<j\right\}$, and still consists of blue open arcs on which $f$ is 1-to-1 and black half open, half closed arcs.

Let $y=\operatorname{lub} f\left(R_{j}\right)$. If $y=1$, let $G_{j}=R_{j}$ and color it green. Otherwise, suppose there are $m$ blue points that map to $y$ as well as the $j-1$ green points that map to $y$. Each of the blue points is in an open interval on which $f$ is 1-to-1, so there is a positive number $e$ such that there are at least $m+1$ blue points that map to $y-e$, including the one in $R_{j}$. The $e$ can also be made small enough that there are still $j-1$ green points that map to $y-e$. Since $f^{-1}(y-e)$ has less than $k+1$ points, $(j-1)+(m+1)<k+1$. Hence there is at least one black point that maps to $y$.

The function $f$ is not 1-to-1 on the black half open, half closed arcs, but the graph of $f$ restricted to a black arc is at each of its points either a crossing point, a local maximum, or a local minimum. This is true of any finite-to- 1 map, and $f$ is continuous on each black, or blue, arc in $M$. For each black point that maps to $y$ that is either a crossing or a local maximum, there is added to $f^{-1}\left(y-e^{\prime}\right)$, for some $e^{\prime}<e$, at least one black point. Hence there is at least one black point, $x$, that maps to $y$ and is a local minimum.

Suppose the half open, half closed arc in $M$ that $x$ belongs to is $[a, b)$. Either there is a first point $c$ in $[a, b)$ between $x$ and $b$ at which $f \uparrow[x, b]$ is a maximum, or lub $f([x, b))=\lim \{f(c) \mid c \rightarrow b\}$ in $[x, b)$. In the former case put $T_{1}=[x, c)$ in $H_{j}$, a precursor of $G_{j}$, and in the latter case put $T_{1}=[x, b)$ in $H_{j}$. The $\operatorname{arc}[a, b)-T_{1} \cdot$ is the union of 0,1 , or 2 half open, half closed arcs; put them back in $M$ and remove $[a, b)$ from $M$. Note that $f$ maps $T_{1}=\left[x, x^{\prime}\right)$ to $\left[y, \operatorname{lub} f\left(T_{1}\right)\right)$, with $f(x)=y$.

Now suppose the half open, half closed arcs $T_{1}, T_{2}, \ldots, T_{\alpha}, \ldots$ have been constructed for all $\alpha<\beta$ so that:

(1) if $T_{\alpha}=\left[x_{\alpha}, x_{\alpha}^{\prime}\right)$, then $f$ satisfies the hypothesis of Lemma 3, i.e. $f\left(T_{\alpha}\right)=$ $\left[f\left(x_{\alpha}\right), \operatorname{lub}\left(f\left(T_{\alpha}\right)\right)\right)$,

(2) each $T_{\alpha}$ is black,

(3) if $f\left(T_{\alpha}\right)=\left[y_{0}, y_{1}\right)$ in $[0,1)$, then there is a number $y_{2}>y_{1}$ such that $f\left(T_{\alpha+1}\right)=\left[y_{1}, y_{2}\right)$, and

(4) if $\alpha$ is a limit ordinal, then there is a number $y$ greater than

$$
y_{0}=\operatorname{lub}\left\{f\left(T_{\gamma}\right) \mid \gamma<\alpha\right\}
$$

such that $f\left(T_{\alpha}\right)$ is $\left[y_{0}, y\right)$.

Now, if $y=\operatorname{lub}\left\{f\left(T_{\alpha}\right) \mid \alpha<\beta\right\}$ is 1 , we are through constructing $H_{j}$. Otherwise, exactly as in the construction of $T_{2}, T_{\beta}$ is formed. The only minor difference is that $f^{-1}(y-e)$ has at least one black point from $\bigcup\left\{T_{\alpha} \mid \alpha<\beta\right\}$, rather than a blue point from $R_{j}$. Continue until $y=1$ is reached, finishing the construction of $H_{j}$.

Each $T_{\alpha}=\left[x_{\alpha}, x_{\alpha}^{\prime}\right)$, for all relevant $\alpha$, maps onto some $\left[y_{\alpha}, y_{\alpha+1}\right)$ in $[0,1]$ with $f\left(x_{\alpha}\right)=y_{\alpha}$ and $y_{\alpha+1}=\operatorname{lub} f\left(T_{\alpha}\right)$. From Lemma 3 , there is a collection $C_{\alpha}$ of disjoint half open, half closed arcs in $T_{\alpha}$ such that $f \uparrow\left(T_{\alpha}-\bigcup C_{\alpha}\right)$ is 1-to-1 and has the same $\left[y_{\alpha}, y_{\alpha+1}\right)$ image. Color each point of $T_{\alpha}-\bigcup C_{\alpha}$ green and put it in the set $G_{j}$ under construction. The half open half closed arcs of $C_{\alpha}$ stay black and are returned to the ever-changing collection $M$.

By induction $G_{1}, G_{2}, \ldots, G_{k}$ are defined and the theorem is proved.

Proof of MAIN Claim. All sets in this proof are presumed to be in $X^{\prime}$. The property that each point in $X^{\prime}$ connects to $D$ via an arc in $X^{\prime}$ is heavily used, as 
are the conclusions of Lemma 0 and Lemma 1, which are true for compact subsets of $X$.

Let $F=B_{1} \cup B_{2} \cup \cdots \cup B_{k+1} \cup D \subset X^{\prime}$. We will find a collection $C$ of disjoint open or half open, half closed arcs whose union is $X^{\prime}-F$. If points were allowed in $C$, it would be easy. The secret is to cover the most complex $\operatorname{arcs}$ in $X^{\prime}-F$ first. Complexity centers on the following definition:

The point $p$ is an offshoot limit point of the set $Q$ if there is an arc $A$ containing $p$ and a null sequence of arcs converging to $p$, each with one endpoint on $A$ and one endpoint in $Q-A$.

The complexity levels are defined as follows: If $x$ is in $X^{\prime}-F$, then

(1) $\operatorname{level}(x) \geq 0$,

(2) level $(x) \geq i$ if $x$ is an offshoot limit point of points whose level is at least $i-1$, and

(3) $\operatorname{level}(x)=i$ if $\operatorname{level}(x) \geq i$ but not $\operatorname{level}(x) \geq i+1$.

Many continua have arbitrarily high levels, but not one on which there is an at most $k$-to-1 function to $(0,1)$ :

Fact 1. No point in $X^{\prime}-F$ has level $k+1$ or higher.

PROOF. Suppose on the contrary that there is a point $p_{1}$ in $X^{\prime}-F$ with level $\left(p_{1}\right) \geq k+1$. By definition there is an arc $S_{1}$ containing $p_{1}$ and a null sequence of offshoot arcs converging to $p_{1}$ with non- $S_{1}$ endpoints in level $k$ or higher. Since $p_{1}$ is not in the closed set $F$, we may assume that $S_{1}$ and all of its offshoot arcs also miss $F$. Let $f\left(S_{1}\right)=\left[a_{1}, b_{1}\right]$ in $A_{1}=(0,1)$.

Now suppose for each $i<j<k+2$ an arc $S_{i}$ in $X^{\prime}-F$ has been chosen and also a point $p_{j}$ in $X^{\prime}-F$ so that:

(1) $S_{n} \cap S_{m}=\varnothing$ if $n \neq m$ and both are less than $j$,

(2) $f\left(S_{m}\right)=\left[a_{m}, b_{m}\right] \subset\left(a_{m-1}, b_{m-1}\right)$ for $1<m<j$,

(3) $p_{j}$ is not in any $S_{i}, i<j$, and

(4) $p_{j}$ has level at least $k+2-j$ and maps into $\left(a_{j-1}, b_{j-1}\right)$.

Note that each point in $\left(a_{j-1}, b_{j-1}\right)$ has an inverse in each of the disjoint $S_{i}$, $i<j$.

By the definition of $(k+2-j)$-level, there is an arc $S_{j}$ in $X^{\prime}-F$ containing $p_{j}$ with an attached null sequence of offshoot arcs converging to $p_{j}$ with endpoints of level at least $k+1-j$. This $S_{j}$ can be made short enough to miss the other $S_{i}$, $i<j$, since $p_{j}$ misses them. Since $p_{j}$ maps into $\left(a_{j-1}, b_{j-1}\right)$ the arc $S_{j}$ can also be made short enough that $f\left(S_{j}\right)=\left[a_{j}, b_{j}\right]$ lies in $\left(a_{j-1}, b_{j-1}\right)$. Thus properties (1) and (2) are still true.

Since at most a finite number of disjoint arcs can map to any interval that contains either $a_{j}$ or $b_{j}$ and since each offshoot arc has one point on $S_{j}$ that maps to $\left[a_{j}, b_{j}\right]$, there is an offshoot endpoint $p_{j+1}$ of level at least $k+1-j$ that maps into $\left(a_{j}, b_{j}\right)$ that is close enough to $p_{j}$ to not be in any $S_{i}, i<j$; it misses $S_{j}$ by definition of offshoot limit point.

Thus the process can continue inductively until $\left[a_{k+1}, b_{k+1}\right]$ has too many point inverses for each of its points. Q.E.D. (for Fact 1)

Fact 2. Suppose $F^{\prime}$ is a closed set in $X^{\prime}$ containing $F$ and suppose $P$ is a closed set in $X^{\prime}-F^{\prime}$ with no offshoot limit points. Then there is a finite collection of arcs in $X^{\prime}$ whose union contains $P$ such that each component of this union intersects $F^{\prime}$. 
ProOF. Well-order $P$. Let $S_{1}$ be an arc from the first point $p_{1}$ of $P$ to $F^{\prime}$. If $p_{a}$ and $S_{a}$ have been selected for each $a<b$, let $p_{b}$ be the first point of $P$ not in $B_{b}=\mathrm{Cl}\left(\bigcup S_{a} \mid a<b\right)$, and let $S_{b}$ be an irreducible arc from $p_{b}$ to $B_{b}$. Finally, let $c$ be the least ordinal greater than each ordinal used, and let $B_{c}=\mathrm{Cl}\left(\bigcup S_{a} \mid a<c\right)$.

Claim. (1) Each $B_{b}$ is the union of finitely many arcs, each component of which intersects $F^{\prime}$, and (2) if $a<b$ then there is a finite set of points in $P, p_{1}, p_{2}, \ldots, p_{n}$, such that $B_{b}$ is $B_{a}$ plus an irreducible arc $T_{1}$ from $p_{1}$ to $F^{\prime} \cup B_{a}$, plus an irreducible arc $T_{2}$ from $p_{2}$ to $F^{\prime} \cup B_{a} \cup T_{1}, \ldots$, plus an irreducible arc $T_{n}$ from $P_{n}$ to $F^{\prime} \cup B_{a} \cup$ $T_{1} \cup \cdots \cup T_{n-1}$. Furthermore, if $b$ is a limit ordinal then there are on each $T_{i}$ points of $P$ arbitrarily close to $p_{i}$.

Let $b$ be the least ordinal such that the claim is false. The first, $B_{2}=S_{1}$, satisfies part (1) by construction and part (2) vacuously. If $b$ has a predecessor, then $B_{b}$ is $B_{b-1}$ plus a new arc $S_{b-1}$ irreducible from $p_{b-1}$ to $F^{\prime} \cup B_{b-1}$, and so satisfies part (2) by induction and part (1) follows from part (2).

Now suppose $b$ is a limit ordinal. First, $b$ cannot be an uncountable ordinal since each $B_{a+1}-B_{a}$ contains an arc. If $b$ is uncountable then there is an uncountable collection of disjoint arcs and an infinite subcollection whose diameters are bound away from 0 (contradicting Lemma 0 ). Since $b$ is countable, then, there is a sequence of increasing ordinals, $b 1, b 2, \ldots$ whose limit is $b$. Let $b 1=2$, the first relevant ordinal.

Consider the structure of these $B_{b i}$. The first, $B_{2}$, is an arc from a point of $P$ to $F^{\prime}$. Then, for $B_{b 2}$, there is a finite subset of $P$ satisfying part (2) of the claim for $b 2$ and $b 1=2$. Consider one of the arcs $T_{j}$ irreducible from $p_{j}$ to $F \cup B_{b 1} \cup T_{1} \cup$ $\cdots \cup T_{j-1}$. The arc $T_{j}$ either goes directly to $F^{\prime}$ or to one of the previous $T_{i}$, or to $S_{1}$.

In the process of building the $B$ 's only finitely many $T$ 's can go to $F^{\prime}$. Since the $T$ 's are disjoint (without their endpoints), any infinite collection is null. So if infinitely many $T$ 's intersected $F^{\prime}$ then some point of $F^{\prime}$ would be a limit of $T$ 's and hence of $P$ since each $T$ has a point of $P$. This contradicts the hypothesis that $F^{\prime}$ and $P$ are disjoint closed sets.

Back to the more prevalent case then: suppose $T_{j}$ intersects a previous $T_{i}$ and its non- $p_{j}$ endpoint is $p_{i}$, the endpoint of $T_{i}$. If $T_{j}$ is the first $T$ past $T_{i}$ to do this then $T_{j}$ merely extends the arc $T_{i}$ and no triod is formed. Otherwise, if $T_{j}$ hits $T_{i}$ at an interior point, or if some previous $T$ extended $T_{i}$ and $T_{j}$ hits $T_{i}$ at $p_{i}$, then a triod is formed whose three endpoints are all in $P$. Another way that a triod can be formed is if some $p_{i}=p_{j}$ at a limit level, or if the limit $p_{i}$ belongs to some previous $T$. Such a point $p_{i}$ is a possible junction point and any triod with $p_{i}$ as junction point either has regular $T$ legs or arbitrarily short legs tipped with points from $P$ from the "furthermore" part of the claim. We will show that there are only finitely many triods formed in this construction, namely:

Subclaim. There are only finitely many $B_{b i}$ that contain triods.

Suppose a single $T_{3}$ from some $B_{b j}$ is abutted by infinitely many later $T$ 's from $B_{b j}$ and later $B$ 's in the sequence. As before, the $T$ 's form a null sequence, and a convergent subsequence converges to some point of $T_{3}$ which is an offshoot limit point of $P$ since each $T$ has a point of $P$. But $P$ has no offshoot limit points.

Now suppose that there are infinitely many triods formed using all different $T$ 's. The triods form a null sequence and some subsequence $t_{1}, t_{2}, \ldots$ converges to the 
point $p_{0}$ in $P$. Since $X$ is locally connected, there is a short arc $C_{1}$ from $p_{0}$ to $t_{1}$, a shorter arc $C_{2}$ from $p_{0}$ to $t_{2}$, etc., so that $\left\{C_{i}\right\}$ converges to $p_{0}$, and none intersect $F^{\prime}$.

For each $i>1$, there is an arc in $t_{i} \cup C_{i}$ from each endpoint of $t_{i}$ to the first arc $C_{1}$. If $p_{0}$ is not to be an offshoot limit point of $P$, all except finitely many of the endpoints of the triods must be in $C_{1}$. We will suppose they all are and we will assume that $p_{0}$ does not belong to any of the triods, since $p_{0}$ only belongs to at most one. The endpoints of $t_{2}$ are in $C_{1}$; replace the subarc of $C_{1}$, between the first point of $C_{1}$ in $t_{2}$ and the last point of $C_{1}$ in $t_{2}$, with the subarc of $t_{2}$ between the same two points. Since no arc in $t_{2}$ contains all three endpoints, one of them is no longer in $C_{1}$. Closer to $p_{0}$ there is another triple of endpoints whose triod misses $t_{2}$ and another similar substitution can be made. After doing this for infinitely many triods, the new arc with its offshoot triod arcs to $P$, makes $p_{0}$ an offshoot limit point of $P$; a contradiction. This establishes the subclaim.

Thus, for some $i$, every new arc that goes to $B_{b i}$ simply extends some previous arc. If $B_{b i}$ has, say, $n$ endpoints, then so does each $B_{b j}$ for $b j>b i$. Each ray produced by tacking one arc to the end of the previous arc has a unique limit point, since the sequence of arcs is null. These $n$ rays, plus their limit points, form $n$ arcs that decompose $B_{b}-B_{b i}$, and there are, as required in the "furthermore" part of the claim (2), points of $P$ arbitrarily close to the limit points of the rays. If $a<b$, then some $b j$ past $b i$ is greater than $a$ and by induction, $B_{b j}-B_{a}$ is a successive arc buildup as required, and by using final segments of the rays (plus their limit points) to structure $B_{b}-B_{b j}$, the difference $B_{b}-B_{a}$ has the structure required to satisfy the rest of part (2) of the claim. Part (1) follows from the fact that $B_{b}$ is the union of $B_{b i}$ and the $n$ arcs of $B_{b}-B_{b i}$.

This establishes the claim, completes the induction, and proves Fact 2.

Note. If $P \subset U$, an open set in $X^{\prime}$ with the property that for each point $x$ in $P$ there is an arc from $x$ to $F^{\prime}$ in $U$, then each arc in the finite collection that satisfies the conclusion of Fact 2 is in $U$ if the original arcs, $S_{a}$, are constructed in $U$ in the beginning.

(Proof of the main claim, continued.) Recall that the aim is to decompose $X^{\prime}-F$ into a collection of disjoint open or half open, half closed arcs.

Let $\left\{e_{i}\right\}$ denote a sequence of positive numbers converging to 0 with the property that if $q$ belongs to the $e_{i}$-neighborhood of $F$, denoted $N_{e i}(F)$, there is an arc from $q$ to $F$ in $N_{e, i-1}(F)$. This sequence exists since $F$ is compact and $X^{\prime}$ is locally connected.

For each $i$, let $P(i)$ denote the $i$-level (of complexity) points in $X^{\prime}-F$. Define $P_{k 0}=P(k)-N_{e 1}(F)$ and for $i>0$, define $P_{k i}=P(k) \cap\left(\mathrm{Cl}\left(N_{e i}(F)-N_{e, i+1}(F)\right)\right)$. Since there are no $(k+1)$-level points outside of $F$ (Fact 1$)$, each $P_{k i}$ is closed, misses $F$, and has no offshoot limit points. Hence from Fact 2 , there is for each $i$, a finite collection $H_{i}$ of arcs such that $P_{k i} \subset \bigcup H_{i}$, and each component of the union of the elements of $H_{i}$ has a point of $F$. If $i>1$, make $\bigcup H_{i} \subset N_{e, i-1}(F)$. (See the note at the end of the proof of Fact 2.)

Let $S_{1}$ be an arc in $H_{0}$ containing a point of $F$. Each component of $S_{1}-F$ is an open or half open, half closed arc. Put in the collection $L_{k 0}$ (being constructed) each component of $S_{1}-F$ that contains a point of $P(k)$. 
Each component of $\bigcup H_{0}$ intersects $F$ so there is a second arc $S_{2}$ in $H_{0}$ (if $\left.H_{0} \neq\left\{S_{1}\right\}\right)$ that intersects $S_{1}$ or $F$. Add to $L_{k 0}$ the open or half open, half closed arc components of $S_{2}-\left(S_{1} \cup F\right)$ that contain points of $P(k)$. For each $e>0$, there are only finitely many $L_{k 0}$ members outside $N_{e}(F)$ since no point outside of $F$ is an offshoot limit point of $P(k)$. Continue this process with the other arcs of $H_{0}$ one at a time. So far:

(1) $\bigcup L_{k 0}$ contains $P_{k 0}$,

(2) $\mathrm{Cl}\left(\bigcup L_{k 0}\right)-\bigcup L_{k 0} \subset F$,

(3) the members of $L_{k 0}$ are disjoint open or half open, half closed arcs that miss $F$, only finitely many of which are more than $e$ away from $F$, for any $e>0$, and

(4) $\bigcup L_{k 0} \subset \bigcup H_{0}$.

Now for $H_{1}$. Some arc $T_{1}$ in $H_{1}$ intersects $F$. Each component of $T_{1}-$ $\left(F \cup\left(\bigcup L_{k 0}\right)\right)$ is an open or half open, half closed arc since $F \cup\left(\bigcup L_{k 0}\right)$ is closed; those components that contain a point of $P(k)$ will be put in $L_{k 1}$. Continue this process on the other members of $H_{1}$, one at a time. Now:

(i) $\left(\bigcup L_{k 0}\right) \cup\left(\bigcup L_{k 1}\right)$ contains $P_{k 0} \cup P_{k 1}$,

(ii) the previous properties (2) and (3) hold with $L_{k 0}$ replaced by $L_{k 0} \cup L_{k 1}$, and (iii) $\bigcup L_{k 1} \subset\left(\bigcup H_{1}\right) \subset N_{e 0}(F)$.

Continue and let $L_{k}=\bigcup\left\{L_{k i} \mid i=0,1, \ldots\right\}$. From (i), $P(k) \subset \bigcup L_{k}$. For each $e>0$, there is an $i$ such that $\bigcup\left\{L_{k j} \mid j>i\right\} \subset N_{e}(F)$, and each of $L_{k 0}, L_{k 1}, \ldots, L_{k i}$ has only finitely many members outside of $N_{e}(F)$. This fact together with property (2) and the fact that any infinite sequence of the disjoint $L_{k}$ is null, ensures that $\mathrm{Cl}\left(\bigcup L_{k}\right)-\bigcup L_{k} \subset F$. The earlier $L_{k i}$ are not changed so property (3) ensures that $L_{k}$ is a collection of disjoint open and half open, half closed arcs that miss $F$.

For $P(k-1)$, use in the place of $F$ the closed set $F^{\prime}=F \cup\left(\bigcup L_{k}\right)$. Then $F^{\prime}$ contains all offshoot limit points of $P(k-1)$ since they are all in $F \cup P(k)$. The same construction yields $L_{k-1}$, a collection of disjoint open or half open, half closed arcs that miss $F^{\prime}$ and whose union contains those points of $P(k-1)$ not already in $\bigcup L_{k}$.

Finally, $L_{k} \cup L_{k-1} \cup \cdots \cup L_{0}$ will decompose all of $X^{\prime}-F$ into disjoint open or half open, half closed arcs. Q.E.D. (Main claim)

\section{REFERENCES}

1. K. Borsuk and R. Molski, On a class of continuous maps, Fund. Math. 45 (1958), 84-98.

2. P. Civin, Two-to-one mappings of manifolds, Duke Math. J. 10 (1943), 49-57.

3. P. Gilbert, $n$-to-one mappings of linear graphs, Duke Math. J. 9 (1942), 475-486.

4. O. G. Harrold, The non-existence of a certain type of continuous transformation, Duke Math. J. 5 (1939), 789-793.

5. __ Exactly $(k, 1)$ transformations on connected linear graphs, Amer. J. Math. 62 (1940), 823-834.

6. J. W. Heath, Every exactly 2-to-1 function on the reals has an infinite number of discontinuities, Proc. Amer. Math. Soc. 98 (1986), 369-373.

7. __ $k$-to-1 functions on arcs for $k$ even, Proc. Amer. Math. Soc. 101 (1987), 387-391.

8. H. Katsuura and K. Kellum, $k$-to-1 functions on an arc, Proc. Amer. Math. Soc. (to appear).

9. K. Kuperberg, Example presented at the Spring Topology Conference, USL, Lafayette, La., March 1986.

10. V. Martin and J. H. Roberts, Two-to-one transformations on 2-manifolds, Trans. Amer. Math. Soc. 49 (1941), 1-17. 
11. J. Mioduszewski, On two-to-one continuous functions, Dissertationes Math. (Rozprawy Mat.) 24 (1961), 42.

12. S. B. Nadler, Jr. and L. E. Ward, Jr., Concerning exactly $(n, 1)$ images of continua, Proc. Amer. Math. Soc. 87 (1983), 351-354.

13. J. H. Roberts, Two-to-one transformations, Duke Math. J. 6 (1940), 256-262.

Division of Mathematics, Foundations, ANalysis and TOPOlOgy, AUbURn UniVersity, Auburn, Alabama 36849-3501 